\title{
Best Possible Maximum Principles for Fully Nonlinear Elliptic Partial Differential Equations
}

\author{
G. Porru, A. Safoui and S. Vernier-Piro
}

\begin{abstract}
We investigate a class of equations including generalized Monge-Ampere equations as well as Weingarten equations and prove a maximum principle for suitable functions involving the solution and its gradient. Since the functions which enjoy the maximum principles are constant for special domains, we have a so called best possible maximum principle that can be used to find accurate estimates for the solution of the corresponding Dirichlet problem. For these equations we also give a variational form which may have its own interest.
\end{abstract}

Keywords. Fully nonlinear elliptic equations, Weingarten surfaces, best possible maximum principles

Mathematics Subject Classification (2000). 35B50, 35J60, 53A10

\section{Introduction}

Let $\Omega \subset \mathbb{R}^{N}, N \geq 2$, be a bounded domain and let $u$ be a smooth function defined in $\Omega$. If $x=\left(x_{1} \ldots, x_{N}\right) \in \Omega$ we put $u_{i}=\frac{\partial u}{\partial x_{i}}, i=1, \cdots, N$, and define $A=\left[u_{i j}\right]$, the (symmetric) Hessian matrix of $u$. For $1 \leq k \leq N$ let

$$
S^{(k)}\left(\lambda_{1}, \ldots, \lambda_{N}\right)
$$

be the $k$-th elementary symmetric function of the eigenvalues of $A$. Famous equations investigated by L. Caffarelli, L. Nirenberg and J. Spruck in [2] are the following:

$$
S^{(k)}\left(\lambda_{1}, \ldots, \lambda_{N}\right)=1
$$

Blow-up solutions of the same equations are discussed by P. Salani in [11]. One can generalize equations (1.1) as follows. Let $g(s)$ be a positive smooth real function satisfying

$$
G(s)=g(s)+2 s g^{\prime}(s)>0 .
$$

G. Porru and S. Vernier-Piro: Dipartimento di Matematica e Informatica, Universitá di Cagliari, 09124 Cagliari, Italy; porru@unica.it, svernier@unica.it

A. Safoui: Department of Mathematics, University of Marrakesh, Marrakesh, Morocco; safoui@ucam.ac.ma 
Define the matrix

$$
Q=I-\frac{2 g^{\prime}\left(|\nabla u|^{2}\right)}{G\left(|\nabla u|^{2}\right)} R, \quad R=\nabla u(\nabla u)^{t},
$$

where $I$ is the $N \times N$ unit matrix and $(\nabla u)^{t}$ is the transposed matrix of $\nabla u$. Note that $Q$ is symmetric and positive definite. One finds

$$
Q^{-1}=I+\frac{2 g^{\prime}\left(|\nabla u|^{2}\right)}{g\left(|\nabla u|^{2}\right)} R .
$$

Using the Hessian matrix $A=\left[u_{i j}\right]$ of $u$, define the new matrix

$$
E=\left[e_{i j}\right]=g\left(|\nabla u|^{2}\right) Q^{-1} A .
$$

By standard results on matrix theory [1], $E$ is similar to a diagonal matrix with entries $\mu_{1}, \ldots, \mu_{N}$, the (real) eigenvalues of $E$. For $1 \leq k \leq N$ let $\sigma^{(k)}\left(\mu_{1}, \ldots, \mu_{N}\right)$ be the $k$-th elementary symmetric function of the eigenvalues of $E$. In this paper we deal with the equations

$$
\sigma^{(k)}\left(\mu_{1}, \ldots, \mu_{N}\right)=1
$$

For $g=1$ we have $E=A$ and equations (1.6) coincide with equations (1.1). For $g=(1+s)^{-\frac{1}{2}}$ we find $Q=I+R$, and we have

$$
E=\left(1+|\nabla u|^{2}\right)^{-\frac{1}{2}} Q^{-1} A
$$

In this situation $\mu_{1}, \ldots, \mu_{N}$ coincide with the principal curvatures of the surface $u=u(x)$ in $\mathbb{R}^{N+1}$, and the corresponding equations (1.6) describe the Weingarten surfaces investigated by L. Caffarelli, L. Nirenberg and J. Spruck in [3].

Let $\alpha \in \mathbb{R}$ with

$$
0 \leq \alpha \leq\left(\begin{array}{c}
N \\
k
\end{array}\right)^{-\frac{1}{k}}
$$

If $G(s)$ is the function introduced in (1.2) and if $\alpha$ satisfies (1.7), we define

$$
\Psi(x)=\frac{1}{2} \int_{0}^{|\nabla u|^{2}} G(s) d s-\alpha u
$$

In Section 3 we prove that if $u$ is a solution to equation (1.6), then the function $\Psi$ cannot assume its maximum value in $\Omega$ unless it is a constant. For $g=1$ and $g(s)=(1+s)^{-\frac{1}{2}}$ this result has been proved in [8] by G. A. Philippin and the second author. We also show that if $\Omega$ is a ball, then we have $\Psi(x)=$ constant, therefore our result is a best possible maximum principle. In Section 4 we give a variational form for the invariants $\sigma^{(k)}\left(\mu_{1}, \ldots, \mu_{N}\right)$. 


\section{Newton tensor}

Let $E$ be a matrix similar to the diagonal matrix $\operatorname{diag}\left\{\mu_{1}, \ldots, \mu_{N}\right\}$. For $k=$ $1, \ldots, N-1$, the $k$-th Newton tensor $T^{(k)}$ is defined as

$$
T^{(k)}=\sigma^{(k)} I-T^{(k-1)} E^{t}, \quad T^{(0)}=I,
$$

where $I$ is the $N \times N$ unit matrix, $E^{t}$ is the transposed of $E$ and $\sigma^{(k)}$ denotes the $k$-th elementary symmetric function of the eigenvalues of $E$. By (2.1) one finds

$$
T^{(k)}=\sigma^{(k)} I-\sigma^{(k-1)} E^{t}+\cdots+(-1)^{k}\left(E^{t}\right)^{k} .
$$

Since $T^{(k)}$ is polynomial in $E^{t}$, it is clear that

$$
T^{(k)} E^{t}=E^{t} T^{(k)} .
$$

A representation of the invariant $\sigma^{(k)}$ in terms of the entries $e_{i j}$ of the matrix $E$ has been given by R. C. Reilly [9]:

$$
\sigma^{(k)}=\frac{1}{k !}\left(\begin{array}{c}
i_{1} \cdots i_{k} \\
j_{1} \cdots j_{k}
\end{array}\right) e_{i_{1} j_{1}} \cdots e_{i_{k} j_{k}},
$$

where the generalized Kronecker symbol $\left(\begin{array}{l}i_{1} \ldots i_{k} \\ j_{1} \ldots j_{k}\end{array}\right)$ has the value 1 (respectively -1 ) if the indexes $i_{1}, \ldots, i_{k}$ are distinct and $\left(j_{1}, \ldots, j_{k}\right)$ is an even (respectively odd) permutation of $\left(i_{1}, \ldots, i_{k}\right)$, otherwise it has value zero. Moreover, the summation convention over repeated indexes from 1 to $N$ is in effect.

We also have a formula for the entries $T_{i j}^{(k)}$ of the Newton tensor [9]

$$
T_{i j}^{(k)}=\frac{1}{k !}\left(\begin{array}{c}
i_{1} \cdots i_{k} i \\
j_{1} \cdots j_{k} j
\end{array}\right) e_{i_{1} j_{1}} \cdots e_{i_{k} j_{k}}
$$

By (2.3) and (2.4) we find

$$
\operatorname{tr}\left\{T^{(k)} E^{t}\right\}=(k+1) \sigma^{(k+1)},
$$

where $\operatorname{tr}\{M\}$ denotes the trace of the matrix $M$. We shall use the equation

$$
T_{i j}^{(k-1)}=\frac{\partial \sigma^{(k)}}{\partial e_{i j}}
$$

which follows by (2.3) and (2.4). 


\section{Maximum principles}

Let $k$ be a fixed integer with $1 \leq k \leq N$, and let $g(s)$ be a smooth positive function satisfying

$$
G(s)=g(s)+2 s g^{\prime}(s)>0
$$

for $s \geq 0$. Typical examples are $g(s)=(1+\gamma s)^{-\frac{1}{2}}, \gamma \geq 0$. For $p \in \mathbb{R}^{N}$ define

$$
Q=I-\frac{2 g^{\prime}\left(|p|^{2}\right)}{G\left(|p|^{2}\right)} R, \quad R=p p^{t}
$$

where $I$ is the $N \times N$ unit matrix, $p$ is used as a column matrix, $p^{t}$ is its transposed. Note that $Q$ is symmetric and positive definite. Indeed, the eigenvalues of $Q$ are 1 (counted $N-1$ times) and $\frac{g}{G}$. We have

$$
Q^{-1}=I+\frac{2 g^{\prime}\left(|p|^{2}\right)}{g\left(|p|^{2}\right)} R
$$

Let $u=u(x)$ be a smooth function defined in a bounded domain $\Omega \subset \mathbb{R}^{N}$. If $A=\left[u_{i j}\right]$ is the Hessian matrix of $u$, define the new matrix

$$
E=E(A, p)=g\left(|p|^{2}\right) Q^{-1} A .
$$

Although $E$ is not, in general, symmetric, it is similar to a diagonal matrix with entries $\mu_{1}, \ldots, \mu_{N}$, the (real) eigenvalues of $E$. If $p=\nabla u$ and $g(s)=(1+s)^{-\frac{1}{2}}$ then $\mu_{1}, \ldots, \mu_{N}$ are the principal curvatures of the surface $u=u(x)$ contained in $\mathbb{R}^{N+1}$.

Let $F(E)=\sigma^{(k)}\left(\mu_{1}, \ldots, \mu_{N}\right), E=\left[e_{i j}\right]$, the $k$-th elementary symmetric function of the eigenvalues of $E$. Since $E$ depends on $A$ and $p$, we introduce the notation $Z(A, p)=F(E)$, and define the associated matrices

$$
A^{*}=\left[\frac{\partial Z}{\partial u_{i j}}\right], \quad E^{*}=\left[\frac{\partial F}{\partial e_{i j}}\right] .
$$

Since $F$ is homogeneous with respect to $e_{i j}$, also $Z$ is homogeneous with respect to $u_{i j}$ and

$$
\operatorname{tr}\left\{A^{*} A\right\}=\operatorname{tr}\left\{E^{*} E^{t}\right\}=k F(E) .
$$

By using the chain rule one finds

$$
A^{*}=g\left(|p|^{2}\right) Q^{-1} E^{*} .
$$

By (2.6) we have

$$
E^{*}=\left[\frac{\partial \sigma^{(k)}}{\partial e_{i j}}\right]=T^{(k-1)},
$$


where $T^{(k-1)}=T^{(k-1)}\left(E^{t}\right)$ is the $(k-1)$-th Newton tensor associated with the matrix $E^{t}$. By (2.2) we have

$$
E^{*} E^{t}=E^{t} E^{*}
$$

We have the following

Lemma 3.1. Let $h \in \mathbb{R}^{N}$ be an arbitrary column vector and let $W=p h^{t}+h p^{t}$. Then,

$$
\frac{\partial Z(A, p)}{\partial p} h=\operatorname{tr}\left\{E^{*} E^{t}\left[\frac{2 g^{\prime}}{G} W Q^{-1}+4\left(\frac{g^{\prime}}{G}\right)^{\prime} p^{t} h R Q^{-1}+\frac{2 g^{\prime}}{g} p^{t} h I\right]\right\} .
$$

Proof. Since

$$
Q(p)=I-\frac{2 g^{\prime}\left(|p|^{2}\right)}{G\left(|p|^{2}\right)} R, \quad R=p p^{t}
$$

we have

$$
\begin{aligned}
Q(p+h) & =I-\frac{2 g^{\prime}\left(|p+h|^{2}\right)}{G\left(|p+h|^{2}\right)}(p+h)(p+h)^{t} \\
& =I-\left(\frac{2 g^{\prime}}{G}+4\left(\frac{g^{\prime}}{G}\right)^{\prime} p^{t} h+\cdots\right)(R+W+\cdots) \\
& =Q(p)-\frac{2 g^{\prime}}{G} W-4\left(\frac{g^{\prime}}{G}\right)^{\prime} p^{t} h R+\cdots \\
& =Q\left[I-\frac{2 g^{\prime}}{G} Q^{-1} W-4\left(\frac{g^{\prime}}{G}\right)^{\prime} p^{t} h Q^{-1} R+\cdots\right]
\end{aligned}
$$

where dots stand for terms of higher order with respect to $|h|$. Hence,

$$
\begin{aligned}
Q^{-1}(p+h) & =\left[I+\frac{2 g^{\prime}}{G} Q^{-1} W+4\left(\frac{g^{\prime}}{G}\right)^{\prime} p^{t} h Q^{-1} R+\cdots\right] Q^{-1} \\
& =Q^{-1}+\frac{2 g^{\prime}}{G} Q^{-1} W Q^{-1}+4\left(\frac{g^{\prime}}{G}\right)^{\prime} p^{t} h Q^{-1} R Q^{-1}+\cdots
\end{aligned}
$$

Using this expansion we find

$$
\begin{aligned}
& E(A, p+h) \\
& =g\left(|p+h|^{2}\right) Q^{-1}(p+h) A \\
& =\left(g+2 g^{\prime} p^{t} h+\cdots\right)\left(Q^{-1} A+\frac{2 g^{\prime}}{G} Q^{-1} W Q^{-1} A+4\left(\frac{g^{\prime}}{G}\right)^{\prime} p^{t} h Q^{-1} R Q^{-1} A+\cdots\right) \\
& =E+\frac{2 g^{\prime}}{G} Q^{-1} W E+4\left(\frac{g^{\prime}}{G}\right)^{\prime} p^{t} h Q^{-1} R E+\frac{2 g^{\prime}}{g} p^{t} h E+\cdots .
\end{aligned}
$$


Using the previous formula we find

$$
\begin{aligned}
Z(A, p & +h) \\
& =F(E(A, p+h)) \\
& =F\left(E+\frac{2 g^{\prime}}{G} Q^{-1} W E+4\left(\frac{g^{\prime}}{G}\right)^{\prime} p^{t} h Q^{-1} R E+\frac{2 g^{\prime}}{g} p^{t} h E+\cdots\right) \\
& =Z(A, p)+\operatorname{tr}\left\{E^{*} E^{t}\left[\frac{2 g^{\prime}}{G} W Q^{-1}+4\left(\frac{g^{\prime}}{G}\right)^{\prime} p^{t} h R Q^{-1}+\frac{2 g^{\prime}}{g} p^{t} h I+\cdots\right]\right\} .
\end{aligned}
$$

The lemma follows.

For $p=\nabla u$ and $h=A \nabla u$ we have $R=\nabla u(\nabla u)^{t}, W=R A+A R$. Using Lemma 3.1 we find

$$
\begin{aligned}
\frac{\partial Z(A, \nabla u)}{\partial(\nabla u)} A \nabla u= & \operatorname{tr}\left\{E^{*} E^{t} \frac{2 g^{\prime}}{G}(R A+A R) Q^{-1}\right\} \\
& +(\nabla u)^{t} A \nabla u \operatorname{tr}\left\{E^{*} E^{t}\left[4\left(\frac{g^{\prime}}{G}\right)^{\prime} R Q^{-1}+\frac{2 g^{\prime}}{g} I\right]\right\} .
\end{aligned}
$$

Using equation (1.3) we find $\frac{2 g^{\prime}}{G}(R A+A R)=2 A-Q A-A Q$. Hence, recalling that $A=\frac{1}{g} Q E=\frac{1}{g} E^{t} Q$, we have

$$
\begin{aligned}
\operatorname{tr}\left\{E^{*} E^{t} \frac{2 g^{\prime}}{G}(R A+A R) Q^{-1}\right\} & =\operatorname{tr}\left\{E^{*} E^{t}\left(2 A Q^{-1}-Q A Q^{-1}-A\right)\right\} \\
& =\frac{1}{g} \operatorname{tr}\left\{E^{*} E^{t}\left(2 E^{t}-Q E^{t}-E^{t} Q\right)\right\} \\
& =\frac{2}{g} \operatorname{tr}\left\{E^{*}\left(E^{t}\right) 2(I-Q)\right\} \\
& =\frac{4 g^{\prime}}{g G} \operatorname{tr}\left\{E^{*}\left(E^{t}\right)^{2} R\right\} .
\end{aligned}
$$

We have used (1.3) and the equation $\operatorname{tr}\left\{E^{*} E^{t} Q E^{t}\right\}=\operatorname{tr}\left\{E^{*}\left(E^{t}\right)^{2} Q\right\}$, true because $E^{*} E^{t}=E^{t} E^{*}$. Therefore,

$$
\begin{aligned}
& \frac{\partial Z(A, \nabla u)}{\partial(\nabla u)} A \nabla u \\
& =\frac{4 g^{\prime}}{g G} \operatorname{tr}\left\{E^{*}\left(E^{t}\right)^{2} R\right\}+(\nabla u)^{t} A \nabla u \operatorname{tr}\left\{E^{*} E^{t}\left[4\left(\frac{g^{\prime}}{G}\right)^{\prime} R Q^{-1}+\frac{2 g^{\prime}}{g} I\right]\right\} .
\end{aligned}
$$

Our main result deals with the following equation:

$$
Z(A, \nabla u)=F(E)=\sigma^{(k)}\left(\mu_{1}, \ldots, \mu_{N}\right)=1 .
$$


The Dirichlet problem for equation (3.7) in case of $g=1$ or $g=(1+s)^{-\frac{1}{2}}$ has been investigated in [2] and [3]. A solution to (3.7) is called admissible if the corresponding matrix $A^{*}=\left[\frac{\partial Z}{\partial u_{i j}}\right]$ is positive definite. We refer to $[2,3,8,11]$ for existence, uniqueness and regularity results.

Theorem 3.2. Let $\alpha$ satisfy (1.7), let $g$ satisfy (3.1) and let $u(x)$ be an admissible solution of the nonlinear elliptic equation (3.7) in a bounded domain $\Omega \subset \mathbb{R}^{N}$. Then the function

$$
\Psi(x)=\frac{1}{2} \int_{0}^{|\nabla u|^{2}} G(s) d s-\alpha u
$$

cannot assume its maximum value in $\Omega$ unless it is a constant.

Proof. Differentiating $\Psi$ we find

$$
\begin{aligned}
\Psi_{i} & =G u_{i \ell} u_{\ell}-\alpha u_{i} \\
\Psi_{i j} & =2 G^{\prime} u_{i \ell} u_{\ell} u_{j s} u_{s}+G u_{i j \ell} u_{\ell}+G u_{i \ell} u_{j \ell}-\alpha u_{i j} .
\end{aligned}
$$

With $B=\left[\Psi_{i j}\right]$ we have

$$
\operatorname{tr}\left\{A^{*} B\right\}=2 G^{\prime} \operatorname{tr}\left\{A^{*} A R A\right\}+G \operatorname{tr}\left\{A^{*} A_{\ell}\right\} u_{\ell}+G \operatorname{tr}\left\{A^{*} A 2\right\}-\alpha \operatorname{tr}\left\{A^{*} A\right\} .
$$

Using the equations (3.2), (3.4) with $p=\nabla u$, and recalling that $Q E=E^{t} Q$ (by $(3.2)$ ) and that $E^{*} E^{t}=E^{t} E^{*}$ (by (3.5)) we find

$$
\operatorname{tr}\left\{A^{*} A 2\right\}=\operatorname{tr}\left\{A^{2} A^{*}\right\}=\frac{1}{g} \operatorname{tr}\left\{E^{t} Q E^{t} E^{*}\right\}=\frac{1}{g} \operatorname{tr}\left\{E^{*}\left(E^{t}\right)^{2} Q\right\} .
$$

Consider first $g^{\prime} \neq 0$. Using (1.3) and (3.11) we find

$$
\begin{aligned}
\operatorname{tr}\left\{A^{*} A R A\right\} & =\frac{G}{2 g^{\prime}} \operatorname{tr}\left\{A^{*} A(I-Q) A\right\} \\
& =\frac{G}{2 g^{\prime}} \operatorname{tr}\left\{A^{*} A 2\right\}-\frac{G}{2 g^{\prime}} \operatorname{tr}\left\{A^{*} A Q A\right\} \\
& =\frac{G}{2 g g^{\prime}}\left[\operatorname{tr}\left\{E^{*}\left(E^{t}\right)^{2} Q\right\}-\operatorname{tr}\left\{Q^{-1} E^{*} E^{t} Q^{2} E^{t} Q\right\}\right] \\
& =\frac{G}{2 g g^{\prime}} \operatorname{tr}\left\{E^{*}\left(E^{t}\right) 2(Q-Q 2)\right\} .
\end{aligned}
$$

Using the last equation and the formula $Q-Q 2=\frac{2 g^{\prime} g}{G 2} R$ we find

$$
\operatorname{tr}\left\{A^{*} A R A\right\}=\frac{1}{G} \operatorname{tr}\left\{E^{*}\left(E^{t}\right)^{2} R\right\} .
$$

If $g^{\prime}=0$ then $G=g, Q=I$ and $E=g A$, therefore (3.12) holds trivially in this situation. 
Differentiating the equation $Z(A, \nabla u)=1$ with respect to $x_{\ell}$ and multiplying by $u_{\ell}$ we find

$$
\frac{\partial Z}{\partial u_{i j}} u_{i j \ell} u_{\ell}+\frac{\partial Z}{\partial u_{i}} u_{i \ell} u_{\ell}=0
$$

or, using matrix notation,

$$
\operatorname{tr}\left\{A^{*} A_{\ell}\right\} u_{\ell}+\frac{\partial Z(A, \nabla u)}{\partial(\nabla u)} A \nabla u=0 .
$$

The latter equation and (3.6) yield

$$
\begin{aligned}
\operatorname{tr}\left\{A^{*} A_{\ell}\right\} u_{\ell}= & -\frac{4 g^{\prime}}{g G} \operatorname{tr}\left\{E^{*}\left(E^{t}\right)^{2} R\right\} \\
& -(\nabla u)^{t} A \nabla u \operatorname{tr}\left\{E^{*} E^{t}\left[4\left(\frac{g^{\prime}}{G}\right)^{\prime} R Q^{-1}+\frac{2 g^{\prime}}{g} I\right]\right\} .
\end{aligned}
$$

Since $F(E)=F\left(e_{i j}\right)$ is homogeneous of degree $k$ with respect to $e_{i j}$, also $Z(A, \nabla u)=Z\left(u_{i j}, u_{i}\right)$ is homogeneous of degree $k$ with respect to $u_{i j}$ and, using equation (3.7) we find

$$
\operatorname{tr}\left\{A^{*} A\right\}=\operatorname{tr}\left\{E^{*} E^{t}\right\}=k \sigma^{(k)}=k .
$$

Insertion of (3.11), (3.12), (3.13) and (3.14) into (3.10) leads to

$$
\begin{aligned}
\operatorname{tr}\left\{A^{*} B\right\}= & \frac{2 G^{\prime}}{G} \operatorname{tr}\left\{E^{*}\left(E^{t}\right)^{2} R\right\}-\frac{4 g^{\prime}}{g} \operatorname{tr}\left\{E^{*}\left(E^{t}\right)^{2} R\right\} \\
& -G(\nabla u)^{t} A \nabla u \operatorname{tr}\left\{E^{*} E^{t}\left[4\left(\frac{g^{\prime}}{G}\right)^{\prime} R Q^{-1}+\frac{2 g^{\prime}}{g} I\right]\right\} \\
& +\frac{G}{g} \operatorname{tr}\left\{E^{*}\left(E^{t}\right)^{2} Q\right\}-\alpha k
\end{aligned}
$$

By (3.9) we get

$$
G(\nabla u)^{t} A \nabla u=\alpha|\nabla u|^{2}+(\nabla u)^{t} \nabla \Psi
$$

and

$$
G A R=\alpha R+\nabla \Psi(\nabla u)^{t}
$$

We claim that

$$
\begin{aligned}
& \frac{2 G^{\prime}}{G} \operatorname{tr}\left\{E^{*}\left(E^{t}\right)^{2} R\right\}-G(\nabla u)^{t} A \nabla u \operatorname{tr}\left\{E^{*} E^{t} 4\left(\frac{g^{\prime}}{G}\right)^{\prime} R Q^{-1}\right\} \\
& =\frac{6 g^{\prime}}{g} \operatorname{tr}\left\{E^{*}\left(E^{t}\right)^{2} R\right\}+C^{t} \nabla \Psi
\end{aligned}
$$


where $C$ is a regular vector field. Note that $E^{t} R=g A Q^{-1} R=G A R, R Q^{-1}=$ $\frac{G}{g} R$. Using the latter equations and (3.16) we have

$$
\begin{aligned}
\left(\frac{2 G^{\prime}}{G}-\frac{6 g^{\prime}}{g}\right) \operatorname{tr}\left\{E^{*}\left(E^{t}\right)^{2} R\right\}-G(\nabla u)^{t} A \nabla u \operatorname{tr}\left\{E^{*} E^{t} 4\left(\frac{g^{\prime}}{G}\right)^{\prime} R Q^{-1}\right\} \\
=2 \frac{G^{\prime} g-3 g^{\prime} G}{G g} \operatorname{tr}\left\{E^{*} E^{t} G A R\right\}-G(\nabla u)^{t} A \nabla u 4\left(\frac{g^{\prime}}{G}\right)^{\prime} \frac{G}{g} \operatorname{tr}\left\{E^{*} E^{t} R\right\} \\
=4|\nabla u|^{2} \frac{g^{\prime \prime} g-3\left(g^{\prime}\right) 2}{G g} \operatorname{tr}\left\{E^{*} E^{t} G A R\right\} \\
-4 \alpha|\nabla u|^{2} \frac{g^{\prime \prime} g-3\left(g^{\prime}\right) 2}{G g} \operatorname{tr}\left\{E^{*} E^{t} R\right\}+C^{t} \nabla \Psi
\end{aligned}
$$

The latter equation and (3.17) yield (3.18).

Insertion of (3.18) into (3.15) and use of (3.16) leads to

$$
\begin{aligned}
\operatorname{tr}\left\{A^{*} B\right\}= & \frac{6 g^{\prime}}{g} \operatorname{tr}\left\{E^{*}\left(E^{t}\right)^{2} R\right\}-\frac{4 g^{\prime}}{g} \operatorname{tr}\left\{E^{*}\left(E^{t}\right)^{2} R\right\}+\frac{G}{g} \operatorname{tr}\left\{E^{*}\left(E^{t}\right)^{2} Q\right\} \\
& -\alpha|\nabla u|^{2} \frac{2 g^{\prime}}{g} \operatorname{tr}\left\{E^{*} E^{t}\right\}-\alpha k+C^{t} \nabla \Psi
\end{aligned}
$$

Since $\frac{2 g^{\prime}}{g} R+\frac{G}{g} Q=\frac{G}{g} I$ and $\operatorname{tr}\left\{E^{*} E^{t}\right\}=k$ we find

$$
\begin{aligned}
\operatorname{tr}\left\{A^{*} B\right\}+C^{t} \nabla \Psi & =\frac{G}{g} \operatorname{tr}\left\{E^{*}\left(E^{t}\right) 2\right\}-\alpha|\nabla u|^{2} \frac{2 g^{\prime}}{g} k-\alpha k \\
& =\frac{G}{g}\left[\operatorname{tr}\left\{E^{*}\left(E^{t}\right) 2\right\}-\alpha k\right] .
\end{aligned}
$$

To evaluate the quantity in the square brackets in (3.19) we recall the Newton tensor relative to $E^{t}$

$$
T^{(k)}=\sigma^{(k)} I-T^{(k-1)} E^{t}, \quad T_{0}=I,
$$

introduced in Section 2. Since $E^{*}=T^{(k-1)}$, by (3.20) we find

$$
E^{*}\left(E^{t}\right) 2=T^{(k-1)}\left(E^{t}\right) 2=\sigma^{(k)} E^{t}-T^{(k)} E^{t} .
$$

By (2.5) we have $\operatorname{tr}\left\{T^{(k)} E^{t}\right\}=(k+1) \sigma^{(k+1)}$. Hence,

$$
\operatorname{tr}\left\{E^{*}\left(E^{t}\right) 2\right\}=\sigma^{(k)} \sigma^{(1)}-(k+1) \sigma^{(k+1)} .
$$

Define $\alpha_{k}=\left(\begin{array}{l}N \\ k\end{array}\right)^{-\frac{1}{k}}$. By standard inequalities (see [11]) we have

$$
\sigma^{(k+1)} \leq\left(\begin{array}{c}
N \\
k+1
\end{array}\right) \alpha_{k}^{k+1}\left(\sigma^{(k)}\right)^{\frac{k+1}{k}}
$$


and

$$
\sigma^{(1)} \geq N \alpha_{k}\left(\sigma^{(k)}\right)^{\frac{1}{k}}
$$

Hence, by (3.21) we find

$$
\begin{aligned}
\operatorname{tr}\left\{E^{*}\left(E^{t}\right) 2\right\} & \geq N \alpha_{k}\left(\sigma^{(k)}\right)^{\frac{k+1}{k}}-(k+1)\left(\begin{array}{c}
N \\
k+1
\end{array}\right) \alpha_{k}^{k+1}\left(\sigma^{(k)}\right)^{\frac{k+1}{k}} \\
& =k \alpha_{k}\left(\sigma^{(k)}\right)^{\frac{k+1}{k}} \\
& =k \alpha_{k}
\end{aligned}
$$

where the equation $\sigma^{(k)}=1$ has been used in the last step. Taking into account this result, from (3.19) we find

$$
A_{i j}^{*} \Psi_{i j}+C^{t} \nabla \Psi=\operatorname{tr}\left\{A^{*} B\right\}+C^{t} \nabla \Psi \geq 0 .
$$

Since the matrix $A^{*}$ is positive definite, the theorem follows by Hopf's first maximum principle.

Applications. If $u$ is an admissible solution of equation (3.7) in a bounded domain $\Omega$, then some estimates follow from Theorem 3.1. First of all, taking $\alpha=0$ it follows that $|\nabla u|$ takes its maximum value on the boundary $\partial \Omega$. Moreover, if $u=0$ on $\partial \Omega$, then $u(x)<0$ in $\Omega$ and, by Theorem 3.1 with $\alpha=\alpha_{k}=\left(\begin{array}{l}N \\ k\end{array}\right)^{-\frac{1}{k}}$ we have

$$
\frac{1}{2} \int_{0}^{|\nabla u|^{2}} G(s) d s-\alpha_{k} u \leq \frac{1}{2} \int_{0}^{|\nabla u|_{M} 2} G(s) d s,
$$

where $|\nabla u|_{M}=\sup _{x \in \partial \Omega}|\nabla u|$. In particular, if $u_{m}=\min _{x \in \Omega} u(x)$, then

$$
-\alpha_{k} u_{m} \leq \frac{1}{2} \int_{0}^{|\nabla u|_{M}^{2}} G(s) d s .
$$

Inequality (3.22) becomes an equality if $\Omega$ is a ball. If $\Omega$ is strictly convex, a bound for $|\nabla u|_{M}$ can be found in terms of the geometry of $\partial \Omega$. We refer to [8] for details.

The radial case. If $u=u(r)$ with $r=|x|$ we have

$$
u_{i}=u^{\prime} \frac{x_{i}}{r}, \quad u_{i j}=u^{\prime \prime} \frac{x_{i} x_{j}}{r 2}+u^{\prime} \frac{r 2 \delta_{i j}-x_{i} x_{j}}{r 3},
$$

where $\delta_{i j}$ is the familiar Kroneker delta. At the point $(r, 0, \cdots, 0)$ we have $A=$ $\operatorname{diag}\left\{u^{\prime \prime}, \frac{u^{\prime}}{r}, \ldots, \frac{u^{\prime}}{r}\right\}, Q^{-1}=\operatorname{diag}\left\{\frac{G}{g}, 1, \ldots, 1\right\}$. Hence, $E=g \operatorname{diag}\left\{\frac{G}{g} u^{\prime \prime}, \frac{u^{\prime}}{r}, \ldots, \frac{u^{\prime}}{r}\right\}$. Since $\sigma^{(k)}$ is invariant under rotations, at a point $x$ with $|x|=r$ we find

$$
\sigma^{(k)}=g^{k}\left[\left(\begin{array}{c}
N-1 \\
k-1
\end{array}\right) \frac{G}{g} u^{\prime \prime}\left(\frac{u^{\prime}}{r}\right)^{k-1}+\left(\begin{array}{c}
N-1 \\
k
\end{array}\right)\left(\frac{u^{\prime}}{r}\right)^{k}\right] .
$$


If $u=u(r)$ is a solution of the ordinary differential equation

$$
g\left(\left(u^{\prime}\right) 2\right) u^{\prime}=r \alpha_{k}, \quad \alpha_{k}=\left(\begin{array}{c}
N \\
k
\end{array}\right)^{-\frac{1}{k}}
$$

we have

$$
G\left(\left(u^{\prime}\right) 2\right) u^{\prime \prime}=\alpha_{k} .
$$

By (3.23) we find $\sigma^{(k)}=1$. The corresponding function $\Psi$ with $\alpha=\alpha_{k}$ reads as

$$
\Psi(r)=\frac{1}{2} \int_{0}^{\left(u^{\prime}\right) 2} G(s) d s-\alpha_{k} u
$$

Using (3.24) we find

$$
\frac{d \Psi}{d r}=G\left(\left(u^{\prime}\right) 2\right) u^{\prime \prime} u^{\prime}-\alpha_{k} u^{\prime}=0 .
$$

We conclude that Theorem 3.1 yields a best possible maximum principle.

\section{Variational equations}

In this section we present a variational form of the invariants $\sigma^{(k)}$. By equation (1.5), for $g=1$ we have $E=A$, and the equation

$$
\frac{1}{k}\left(T_{i j}^{(k-1)}(A) u_{j}\right)_{i}=S^{(k)}(A)
$$

is well known. In case of $g(s)=(1+s)^{-\frac{1}{2}}$ the following formula is proved in [10, p. 381]:

$$
\frac{1}{k}\left(\frac{1}{\left(1+|\nabla u|^{2}\right)^{\frac{1}{2}}} T_{i j}^{(k-1)}(E) u_{j}\right)_{i}=\sigma^{(k)}(E) .
$$

We prove a similar formula for a general $g$ by using the Newton tensor $T^{(k-1)}(A)$ instead of $T^{(k-1)}(E)$.

Proposition 4.1. We have

$$
\frac{1}{k}\left(g^{k} T_{i j}^{(k-1)}(A) u_{j}\right)_{i}=\sigma^{(k)}(E)
$$

where $g=g\left(|\nabla u|^{2}\right)$. 
Proof. In this proof the Newton tensors $T^{(k)}(A)$ are relative to $A$ and we write $T^{(k)}$ instead of $T^{(k)}(A)$. As usual we denote by $S^{(k)}$ the $k$-th elementary symmetric function of the eigenvalues of the Hessian matrix $A$.

Let us develop the left hand side of (4.2). Since the Newton tensor $T^{(k-1)}$ is divergence free we have

$$
\frac{1}{k}\left(g^{k} T_{i j}^{(k-1)} u_{j}\right)_{i}=g^{k-1} 2 g^{\prime} T_{i j}^{(k-1)} u_{i \ell} u_{\ell} u_{j}+\frac{1}{k} g^{k} T_{i j}^{(k-1)} u_{i j} .
$$

Recall the Newton equation $T^{(k)}=S^{(k)} I-T^{(k-1)} A$, or

$$
T^{(k-1)} A=S^{(k)} I-T^{(k)} .
$$

Insertion of (4.4) into (4.3) and use of the equation $T_{i j}^{(k-1)} u_{i j}=k S^{(k)}$ yields

$$
\frac{1}{k}\left(g^{k} T_{i j}^{(k-1)} u_{j}\right)_{i}=g^{k-1} 2 g^{\prime}\left[S^{(k)}|\nabla u|^{2}-T_{i j}^{(k)} u_{i} u_{j}\right]+g^{k} S^{(k)} .
$$

Now we develop the right hand side of (4.2). We have

$$
\sigma^{(k)}(E)=\sigma^{(k)}\left(g Q^{-1} A\right)=g^{k} \sigma^{(k)}\left[\left(I+\frac{2 g^{\prime}}{g} R\right) A\right] .
$$

The $(i j)$ entry of the matrix $\left(I+\frac{2 g^{\prime}}{g} R\right) A$ is $u_{i j}+\frac{2 g^{\prime}}{g} u_{j \ell} u_{\ell} u_{i}$. Therefore, using Reilly's formula (2.3) we have

$$
\begin{aligned}
\sigma^{(k)} & {\left[\left(I+\frac{2 g^{\prime}}{g} R\right) A\right] } \\
& =\frac{1}{k !}\left(\begin{array}{c}
i_{1} \cdots i_{k} \\
j_{1} \cdots j_{k}
\end{array}\right)\left(u_{i_{1} j_{1}}+\frac{2 g^{\prime}}{g} u_{j_{1} \ell_{1}} u_{\ell_{1}} u_{i_{1}}\right) \cdots\left(u_{i_{k} j_{k}}+\frac{2 g^{\prime}}{g} u_{j_{k} \ell_{k}} u_{\ell_{k}} u_{i_{k}}\right) .
\end{aligned}
$$

When we develop the product in above we find the term

$$
\frac{1}{k !}\left(\begin{array}{c}
i_{1} \cdots i_{k} \\
j_{1} \cdots j_{k}
\end{array}\right) u_{i_{1} j_{1}} \cdots u_{i_{k} j_{k}}=S^{(k)}
$$

Moreover we find $k$ terms of the kind

$$
\frac{2 g^{\prime}}{g} \frac{1}{k !}\left(\begin{array}{c}
i_{1} \cdots i_{k-1} i \\
j_{1} \cdots j_{k-1} j
\end{array}\right) u_{i_{1} j_{1}} \cdots u_{i_{k-1} j_{k-1}} u_{j \ell} u_{\ell} u_{i} .
$$

We can write the above $k$ terms globally as $\frac{2 g^{\prime}}{g} T_{i j}^{(k-1)} u_{j \ell} u_{\ell} u_{i}$. Using (4.4), the above expression reads as

$$
\frac{2 g^{\prime}}{g}\left[S^{(k)}|\nabla u|^{2}-T_{i j}^{(k)} u_{i} u_{j}\right]
$$


All the remaining terms appearing in the product (4.7) vanish because of the skew-symmetry of the generalized Kronecker symbols. Let us consider in detail the expression

$$
\frac{\left(2 g^{\prime}\right) 2}{g 2} \frac{1}{k !}\left(\begin{array}{c}
i_{1} \cdots i_{k-2} i m \\
j_{1} \cdots j_{k-2} j n
\end{array}\right) u_{i_{1} j_{1}} \cdots u_{i_{k-2} j_{k-2}} u_{j \ell} u_{\ell} u_{i} u_{n s} u_{s} u_{m} .
$$

The term corresponding to $\left(\begin{array}{l}i_{1} \cdots i_{k-2} i m \\ j_{1} \cdots j_{k-2} j n\end{array}\right) u_{i} u_{m}$ cancels the term corresponding to $\left(\begin{array}{l}i_{1} \cdots i_{k-2} i m \\ j_{1} \cdots j_{k-2} n j\end{array}\right) u_{m} u_{i}$. Hence, the insertion of (4.8) and (4.9) into (4.7) yields

$$
\sigma^{(k)}\left[\left(I+\frac{2 g^{\prime}}{g} R\right) A\right]=S^{(k)}+\frac{2 g^{\prime}}{g}\left[S^{(k)}|\nabla u|^{2}-T_{i j}^{(k)} u_{i} u_{j}\right] .
$$

Finally, insertion of (4.10) into (4.6) leads to

$$
\begin{aligned}
\sigma^{(k)}(E) & =g^{k}\left[S^{(k)}+\frac{2 g^{\prime}}{g}\left[S^{(k)}|\nabla u|^{2}-T_{i j}^{(k)} u_{i} u_{j}\right]\right] \\
& =g^{k} S^{(k)}+g^{k-1} 2 g^{\prime}\left[S^{(k)}|\nabla u|^{2}-T_{i j}^{(k)} u_{i} u_{j}\right] .
\end{aligned}
$$

Comparing (4.11) with (4.5), the proposition follows.

\section{References}

[1] Beckenbach, E. F. and Bellman, R., Inequalities. New York: Springer 1993.

[2] Caffarelli, L., Nirenberg, L. and Spruck, J., The Diriclet problem for nonlinear second order elliptic equations, III: Functions of eigenvalues of the Hessian. Acta Math. 155 (1985), 261 - 301.

[3] Caffarelli, L., Nirenberg, L. and Spruck, J., Nonlinear second order elliptic equations, V: The Dirichlet problem for Weingarten hypersurfaces. Comm. Pure Appl. Math. 41 (1988), $47-70$.

[4] Ma X., Isoperimetric bounds for a class of Monge-Ampere equations in two dimensions (to appear in: Analysis).

[5] Gilbarg, D. and Trudinger, N. S., Elliptic Partial Differential Equations of Second Order. Berlin: Springer 1977.

[6] Payne, L. E. and Philippin, G. A., Some maximum principles for nonlinear elliptic equations in divergence form with applications to capillary surfaces and to surfaces of constant mean curvature. Nonlinear Analysis 3 (1979), $193-211$.

[7] Philippin, G. A. and Safoui, A., Some maximum principles and symmetry results for a class of boundary value problems involving the Monge-Ampere equation. Math. Models and Meth. in Appl. Sci. 11 (2001), 1073 - 1080. 
[8] Philippin, G. A. and Safoui, A., Some application of the maximum principle to a variety of fully nonlinear elliptic PDE's. Z. Angew. Math. Phys. 54 (2003), $739-755$.

[9] Reilly, R. C., Variational properties of functions of the mean curvatures for hypersurfaces in space forms. J. Diff. Geometry 8 (1973), $465-477$.

[10] Reilly, R. C., On the Hessian of a function and the curvatures of its graph. Michigan Math. J. 20 (1973), 373 - 383.

[11] Salani, P., Boundary blow-up problems for Hessian equations. Manuscripta Math. 96 (1998), $281-294$.

Received May 27, 2004 